\title{
On the Spherical Symmetry of Static Stellar Models
}

\author{
Lee Lindblom, ${ }^{1,2}$ A.K.M. Masood-ul-Alam ${ }^{1,3}$ \\ 1 Physics Department, Montana State University, Bozeman, Montana 59717, USA \\ 2 Theoretical Astrophysics, California Institute of Technology, Pasadena, California 91125, USA \\ 3 Institute of Theoretical Science, University of Oregon, Eugene, Oregon 97403, USA
}

Received: 11 January 1993/in revised form: 20 September 1993

\begin{abstract}
This paper completes the proof of the necessity of spherical symmetry in the static general-relativistic stellar models that have equations of state satisfying certain inequalities. The technical assumption - that there exists a "reference spherical stellar model" - that was essential in the previous discussions of this problem is removed. This paper also extends beyond previous discussions the class of equations of state included in the proof. The analysis of the equations for spherical stellar models, used here to demonstrate the existence of a "reference spherical model," may also be of independent interest.
\end{abstract}

\section{Introduction}

It seems almost self evident that spherical symmetry is a necessary feature of any equilibrium stellar model which is nonrotating, self gravitating, physically isolated, and composed entirely of fluid. The proof of this "obvious" fact for Newtonian stellar models - although far from trivial - was given many years ago (Lichtenstein [1] or for a more modern discussion Lindblom [2]). The proof for general-relativistic stellar models has been more illusive and is still incomplete. Significant progress has been made recently, however, toward a proof in the relativistic case by Masood-ul-Alam [3,4], Lindblom [5], Beig and Simon [6,7], and Lindblom and Masood-ul-Alam [8]. These discussions show (under various assumptions) that the spatial geometry of static stellar models must be conformally flat as a consequence of the positive mass theorem. Since spatial conformal flatness is equivalent to spherical symmetry in static stellar models (Lindblom [9]), these arguments would be complete proofs of the spherical symmetry conjecture if they did not rely on unphysical assumptions. The purpose of this paper is to remove one of these "technical" assumptions and to weaken the unphysical restrictions on the equation of state of the fluid. We present the first complete proof of the necessity of spherical symmetry for static relativistic stellar models that are composed of a fluid whose adiabatic index satisfies certain inequalities. 
A stellar model in general relativity theory is an asymptotically-flat spacetime that satisfies Einstein's equation with a perfect-fluid source. A static (i.e., time independent and non-rotating) stellar model has a time-translation symmetry whose trajectories are hypersurface orthogonal. Thus, the metric tensor in these spacetimes may always be represented as

$$
d s^{2}=-V^{2} d t^{2}+g_{a b} d x^{a} d x^{b}
$$

where $V$ and the three-dimensional spatial metric $g_{a b}$ are independent of $t$. For simplicity we assume that $V$ and $g_{a b}$ are $C^{3}$ (except at the surface of the star as discussed below). This degree of smoothness guarantees that the strong form of the Bianchi identities may be used everywhere except at the surface of the star. (For a discussion of static stellar models having weaker differentiability conditions see Masood-ul-Alam [10].) Einstein's equation for static stellar models reduces in this representation to the pair of equations

$$
\begin{gathered}
D^{a} D_{a} V=4 \pi V(\varrho+3 p) \\
R_{a b}=V^{-1} D_{a} D_{b} V+4 \pi(\varrho-p) g_{a b} .
\end{gathered}
$$

The density and pressure of the fluid are denoted $\varrho$ and $p$; and the density is assumed to be a given function of the pressure, $\varrho=\varrho(p)$, referred to as the equation of state. This function is assumed to be positive, non-decreasing, and $C^{1}$ for all $p>0$. The spatial covariant derivative compatible with $g_{a b}$ is denoted $D_{a}$, and its Ricci curvature is denoted $R_{a b}$. The Bianchi identity for the three-dimensional spatial geometry may be reduced to

$$
D_{a} p=-V^{-1}(\varrho+p) D_{a} V
$$

with the use of Eqs. (2), (3) for these static fluids.

The solutions of Eqs. (2) and (3) that represent realistic stellar models are those in which the fluid is physically isolated, and so the geometry is taken to be asymptotically flat. The appropriate asymptotic forms for the metric in this case are

$$
\begin{gathered}
V=1-\frac{M}{r}+O\left(r^{-2}\right), \\
g_{a b}^{\cdot}=\left(1+\frac{2 M}{r}\right) \delta_{a b}+O\left(r^{-2}\right),
\end{gathered}
$$

where $M$ is the mass of the star, $\delta_{a b}$ is the flat euclidean metric, and $r$ is a spherical coordinate associated with $\delta_{a b}$. These conditions can be deduced from rather weak assumptions about the asymptotic structure of these spacetimes (see e.g., Beig [11], and Masood-ul-Alam [12]). For simplicity we merely assume here that the spacetime of the static stellar model satisfies Eqs. (5) and (6).

A certain amount of care must be taken to insure that the boundary $V=V_{S}$ between the interior fluid region of the star and the exterior vacuum region is nonsingular. If the density $\varrho$ is discontinuous at the surface of the star, $\varrho(0) \neq 0$, then $V$ and the metric $g_{a b}$ will only be $C^{1,1}$ across this boundary. The most important specific consequence of the continuity conditions at the surface of the star (for our purposes here) is the requirement that the function $W$ defined by

$$
W=D_{a} V D^{a} V
$$

is continuous across the surface of the star, but its derivative satisfies the discontinuity condition

$$
\lim _{V \uparrow V_{S}} n^{a} D_{a} W=\lim _{V \downarrow V_{S}} n^{a} D_{a} W+8 \pi \lim _{V \uparrow V_{S}} V W^{1 / 2} \varrho
$$


where $n^{a}$ is the unit normal to the surface which points out of the interior of the star. These boundary conditions have been discussed in detail in many places (see, e.g., Lindblom [9], or Masood-ul-Alam [4]).

In the following sections we present a proof of the necessity of spherical symmetry for a large class of static stellar models, i.e., solutions of Eqs. (2)-(6). Our proof follows the general outline introduced by Masood-ul-Alam [3] (see also Bunting and Masood-ul-Alam [13]): A conformal factor is found which transforms the spatial metric $g_{a b}$ into a geometry that has vanishing asymptotic mass and non-negative scalar curvature. The positive mass theorem is then used to conclude that the physical geometry is conformally flat. It follows from the analysis of Lindblom [9] that the stellar model must therefore be spherical.

All of the technical difficulties in this method of proof are associated with finding an appropriate conformal factor and demonstrating that it has the desired properties. As in the previous studies of this problem [3-8] we choose as our conformal factor a function which transforms the metric of a "reference spherical model" into the flat euclidean metric. This "reference spherical model" is a spherically symmetric solution to Eqs. (2)-(6) having the same equation of state and the same value of the surface potential $V=V_{S}$ as the given static stellar model. Most of the analysis in this paper is concerned with the demonstration that a suitable "reference spherical model" actually exists. Since solutions to the spherically symmetric equations do not exist for every value of $V_{S}$ (see e.g., Lindblom [15]) our analysis is an important improvement over previous discussions $[4,6,7]$ which merely assume the existence of a suitable "reference spherical model." In Sects. 2 and 3 of this paper we analyze the solutions of the spherically symmetric equations. We demonstrate existence, derive a variety of needed smoothness and monotonicity results, and classify the singularities that arise in the equations. The critical results in these sections are Lemmas 6-8. These show that solutions to the spherical equations exist (for appropriate values of the mass parameter) which can be used as upper and lower estimates for the physical (not necessarily spherical) static stellar model. In Sect. 4 we introduce the constraints on the equation of state that we need to complete the proof of spherical symmetry. These constraints (see Sect. 4 for details) are inequalities which restrict the adiabatic index of the fluid and its first derivative. These restrictions are fairly weak in the sense that there is an open set in the space of all equations of state which satisfy our conditions. Our restrictions are weaker than those introduced by Beig and Simon [6, 7]. In Sect. 5 we show that an appropriate "reference spherical model" exists by constructing the particular solution to the spherically symmetric equations having all the needed properties. The existence of this model follows from the continuity conditions established in Lemma 4, and the upper and lower estimates constructed in Lemmas 6-8. In Sect. 6 we construct the appropriate conformal factor from the "reference spherical model" and derive some of its properties. In Sect. 7 we combine all of the above to show that the chosen conformal factor transforms the physical metric $g_{a b}$ into one that has vanishing asymptotic mass and non-negative scalar curvature. Spatial conformal flatness, and hence spherical symmetry, follows then as a consequence of the positive mass theorem. In Sect. 8 we suggest some directions that future research on strengthening these results might take.

\section{The Functions $W_{\mu}(V)$ and Their Basic Properties}

Consider a static stellar model with finite radius so that the potential $V$ has the value $V_{S}<1$ on the surface of the star. Given the equation of state for the fluid in this 
model, the Bianchi identity Eq. (4) can be integrated with the boundary condition $p\left(V_{S}\right)=0$ :

$$
h(p) \equiv \int_{0}^{p} \frac{d p^{\prime}}{\varrho\left(p^{\prime}\right)+p^{\prime}}=\log \left(\frac{V_{S}}{V}\right)
$$

This expression determines $\varrho$ and $p$ as explicit functions of $V: p(V)=h^{-1}\left[\log \left(V_{S} / V\right)\right]$ and $\varrho(V)=\varrho[p(V)]$. Since we have assumed that the equation of state $\varrho(p)$ is $C^{1}$ for $p>0$, it follows that $\varrho(V)$ and $p(V)$ are $C^{1}$ for $V<V_{S}$. The functions $\varrho(V)$ and $p(V)$ will not in general be differentiable at $V=V_{S}$. These functions admit $C^{0}$ extensions to $V \geq V_{S}$ since $\varrho(p)$ is positive and monotonic for $p>0$. (The extension of $p(V)$ can also be Lipshitz.) Given these functions, we define $r_{\mu}=r_{\mu}(V)$ and $m_{\mu}=m_{\mu}(V)$ to be the solutions of the equations,

$$
\begin{aligned}
\frac{d r_{\mu}}{d V} & =\frac{r_{\mu}\left(r_{\mu}-2 m_{\mu}\right)}{V\left(m_{\mu}+4 \pi r_{\mu}^{3} p\right)}, \\
\frac{d m_{\mu}}{d V} & =\frac{4 \pi r_{\mu}^{3}\left(r_{\mu}-2 m_{\mu}\right) \varrho}{V\left(m_{\mu}+4 \pi r_{\mu}^{3} p\right)},
\end{aligned}
$$

which satisfy the boundary conditions

$$
\begin{gathered}
m_{\mu}\left(V_{S}\right)=\mu, \\
r_{\mu}\left(V_{S}\right)=R_{\mu} \equiv 2 \mu /\left(1-V_{S}^{2}\right),
\end{gathered}
$$

for a given value of the constant $\mu>0$. On the domain where the solutions to Eqs. (10) and (11) exist, we introduce the function

$$
W_{\mu}(V) \equiv\left(1-\frac{2 m_{\mu}}{r_{\mu}}\right)\left(\frac{d r_{\mu}}{d V}\right)^{-2}=\frac{V^{2}\left(m_{\mu}+4 \pi r_{\mu}^{3} p\right)^{2}}{r_{\mu}^{3}\left(r_{\mu}-2 m_{\mu}\right)}
$$

which plays an important role in our proof of the necessity of spherical symmetry. We first determine the domain on which these functions are well defined.

Lemma 1. The solutions to Eqs. (10) and (11), $r_{\mu}(V)$ and $m_{\mu}(V)$, exist on the maximal interval $\left(V_{\mu}, V_{S}\right]$, where $p(V)$ is finite and $W_{\mu}(V)>0$. On this domain $r_{\mu}>0$, $r_{\mu}>2 m_{\mu}$, and $m_{\mu}>-4 \pi r_{\mu}^{3} p$; and if $\lim _{V \downarrow V_{\mu}} p=p_{\mu}<\infty$, then $\sup _{\left(V_{\mu}, V_{S}\right)}\left(2 m_{\mu} / r_{\mu}\right)<1$
and $\lim _{V \downarrow V_{\mu}} W_{\mu}=0$.

Proof. At the surface of the star $r_{\mu}=R_{\mu}=2 \mu /\left(1-V_{S}^{2}\right)>2 \mu=2 m_{\mu}>$ $-8 \pi R_{\mu}^{3} p\left(V_{S}\right)=0$, and the derivatives $d r_{\mu} / d V=V_{S} R_{\mu}^{2} / \mu$ and $d m_{\mu} / d V=$ $4 \pi \varrho\left(V_{S}\right) V_{S} R_{\mu}^{2} / \mu$ are bounded. Therefore solutions to Eqs. (10) and (11) with these boundary conditions exist locally. The needed local existence theorem (see e.g., [14, Theorem 1.1, p. 8]) requires only that the right-hand sides of Eqs. (10) and (11) be continuous functions of $V$. Thus, the local existence of the functions $r_{\mu}$ and $m_{\mu}$ is guaranteed even when $\varrho(V)$ and $p(V)$ are not differentiable at $V=V_{S}$. Consider the maximal interval $\left(V_{\mu}^{\prime}, V_{S}\right.$ ], where the solutions to Eqs. (10) and (11) exist, where $p(V)$ is finite, and where $r_{\mu}>0, r_{\mu}>2 m_{\mu}$ and $m_{\mu}>-4 \pi r_{\mu}^{3} p$. Using Eq. (14) it follows that $W_{\mu}>0$ on $\left(V_{\mu}^{\prime}, V_{S}\right.$ ]. On this interval $d r_{\mu} / d V>0$ so $r_{\mu}$ is monotonic and bounded by $R_{\mu} \geq r_{\mu}>0$. Similarly since $d m_{\mu} / d V>0$ it follows that $m_{\mu}$ 
is monotonic and bounded by $\mu \geq m_{\mu}>-4 \pi r_{\mu}^{3} p$. We also note that since $r_{\mu}$ is monotonic on this domain, we may re-express Eq. (11) in the form

$$
\frac{d m_{\mu}}{d r_{\mu}}=4 \pi r_{\mu}^{2} \varrho \text {. }
$$

If $\lim _{V \downarrow V_{\mu}^{\prime}} p=\infty$ then the lemma follows simply by noting that $V_{\mu}=V_{\mu}^{\prime}$. In the following then, we assume that $\lim _{V \downarrow V_{\mu}^{\prime}} p \equiv p_{\mu}<\infty$. We next show that $\sup _{V_{\mu}}\left(2 m_{\mu} / r_{\mu}\right)<1$ : On the interval $\left(V_{\mu}^{\prime}, V_{S}\right.$ ] the function $W_{\mu}$ is well defined $\left(V_{\mu}^{\prime}, V_{S}\right)$

by Eq. (14), and from Eqs. (10) and (11) it must satisfy the differential equation

$$
\frac{d W_{\mu}}{d V}+\frac{4 W_{\mu}}{r_{\mu}} \frac{d r_{\mu}}{d V}=8 \pi V(\varrho+3 p) .
$$

Equation (16) may be integrated on $\left(V_{\mu}^{\prime}, V_{S}\right]$ to obtain

$$
W_{\mu}(V) r_{\mu}^{4}(V)=\mu^{2}-\int_{V}^{V_{S}} 8 \pi \widehat{V}[\varrho(\widehat{V})+3 p(\widehat{V})] r_{\mu}^{4}(\widehat{V}) d \widehat{V}
$$

This demonstrates that $W_{\mu} r_{\mu}^{4}$ is bounded on $\left(V_{\mu}^{\prime}, V_{S}\right]: \mu^{2} \geq W_{\mu} r_{\mu}^{4}>0$. Using Eq. (14) we may re-express $W_{\mu} r_{\mu}^{4}$ in the form

$$
W_{\mu} r_{\mu}^{4}=\frac{\left(m_{\mu}+4 \pi r_{\mu}^{3} p\right)^{2}}{1-2 m_{\mu} / r_{\mu}} V^{2}
$$

By definition $r_{\mu}>2 m_{\mu}$ on $\left(V_{\mu}^{\prime}, V_{S}\right.$, so we must have $\sup _{\left(V_{\mu}^{\prime}, V_{S}\right)}\left(2 m_{\mu} / r_{\mu}\right)<1$ unless $\lim _{V \downarrow V_{\mu}^{\prime}}\left(2 m_{\mu} / r_{\mu}\right)=1$. But $W_{\mu} r_{\mu}^{4}$ is bounded so in this case the numerator in Eq. (18) would necessarily vanish in this limit. This would imply

$$
\lim _{V \downarrow V_{\mu}^{\prime}} m_{\mu}=-4 \pi \lim _{V \downarrow V_{\mu}^{\prime}} r_{\mu}^{3} p
$$

If $\lim _{V \downarrow V_{\mu}^{\prime}} r_{\mu}>0$, then Eq. (19) implies that

$$
\lim _{V \downarrow V_{\mu}^{\prime}} \frac{2 m_{\mu}}{r_{\mu}}=-8 \pi \lim _{V \downarrow V_{\mu}^{\prime}} r_{\mu}^{2} p<0,
$$

If $\lim _{V \downarrow V_{\mu}^{\prime}} r_{\mu}=0$ we may use l'Hospital's rule and Eq. (15) to conclude that $\lim _{V \downarrow V_{\mu}^{\prime}}\left(2 m_{\mu} / r_{\mu}\right)=0$. In either case we conclude that

$$
\lim _{V \downarrow V_{\mu}^{\prime}} \frac{2 m_{\mu}}{r_{\mu}} \leq 0
$$

But this contradicts our assumption that $\lim _{V \downarrow V_{\mu}^{\prime}}\left(2 m_{\mu} / r_{\mu}\right)=1$, so we conclude that
sup $\left(2 m_{\mu} / r_{\mu}\right)<1$. $\left(V_{\mu}^{\prime}, V_{S}\right)$ 
The final step in the proof of Lemma 1 is to show that $\lim _{V \downarrow V_{\mu}^{\prime}} W_{\mu}=0$, and so conclude that $V_{\mu}=V_{\mu}^{\prime}$. We consider first the case when $\lim _{V \downarrow V_{\mu}^{\prime}} r_{\mu}=0$. It is helpful for
this case to re-write Eq. (14) in the form,

$$
W_{\mu}(V)=V^{2}\left(\frac{m_{\mu}}{r_{\mu}^{2}}+4 \pi r_{\mu} p\right)^{2}\left(1-\frac{2 m_{\mu}}{r_{\mu}}\right)^{-1}
$$

Using l'Hospital's rule and Eq. (15) we conclude that $\lim _{V \downarrow V_{\mu}^{\prime}}\left(m_{\mu} / r_{\mu}^{2}\right)=0$ in this case. Taking the limit of Eq. (22) we conclude then that $\lim _{V \downarrow V_{\mu}^{\prime}} W_{\mu}=0$. This implies that $V_{\mu}=V_{\mu}^{\prime}$, and so the lemma follows for the case $\lim _{V \downarrow V_{\mu}^{\prime}} r_{\mu}=0$.

We turn now to the final case $\lim _{V \downarrow V_{\mu}^{\prime}} r_{\mu}>0$. The functions $m_{\mu}$ and $r_{\mu}$ are absolutely bounded in this case: $\sup _{\left(V_{\mu}^{\prime}, V_{S}\right)}\left|m_{\mu}\right|<\mu+4 \pi R_{\mu}^{3} p_{\mu}$ and $\sup _{\left(V_{\mu}^{\prime}, V_{S}\right)}\left|r_{\mu}\right|=R_{\mu}$. A bound on the derivative of $r_{\mu}$ may be obtained by re-expressing Eq. (10) in the form:

$$
\frac{d r_{\mu}}{d V}=\frac{1}{W_{\mu}^{1 / 2}}\left(1-\frac{2 m_{\mu}}{r_{\mu}}\right)^{1 / 2}
$$

Since $1>2 m_{\mu} / r_{\mu}>-8 \pi r_{\mu}^{2} p$ on the interval $\left(V_{\mu}^{\prime}, V_{S}\right]$, it follows that the derivative of $r_{\mu}$ is bounded absolutely by

$$
\left|\frac{d r_{\mu}}{d V}\right|<\left(\frac{1+4 \pi R_{\mu}^{2} p_{\mu}}{W_{\mu}}\right)^{1 / 2} .
$$

Using Eq. (15) a similar expression may be obtained which bounds the derivative of $m_{\mu}$. It follows then that the derivatives of $r_{\mu}$ and $m_{\mu}$ would be bounded at $V=V_{\mu}^{\prime}$ if $\lim _{V \downarrow V_{\mu}^{\prime}} W_{\mu}>0$. If this were the case the solutions of Eqs. (10) and (11) could be extended beyond $V=V_{\mu}^{\prime}$. Further, in this final case $r_{\mu}>0, r_{\mu}>2 m_{\mu}$, $m_{\mu}>-4 \pi r_{\mu}^{3} p$, and $p=p_{\mu}<\infty$ at $V=V_{\mu}^{\prime}$. By continuity these inequalities would be satisfied by the extended $r_{\mu}$ and $m_{\mu}$ in some neighborhood of $V_{\mu}^{\prime}$. But this violates the assumption that $\left(V_{\mu}^{\prime}, V_{S}\right.$ ] is the maximal interval on which such solutions to Eqs. (10) and (11) exist. Thus, we have a contradiction unless $\lim _{V \downarrow V_{\mu}^{\prime}} W_{\mu}=0$. It follows
then that $V_{\mu}^{\prime}=V_{\mu}$ and the lemma is established. $\square$

We next prove a useful result about the monotonicity of certain combinations of these functions:

Lemma 2. The expression $m_{\mu}-4 \pi \varrho r_{\mu}^{3} / 3$ is a non-decreasing function of $V$ on $\left(V_{\mu}, V_{S}\right]$. And, if the function $d W_{\mu} / d V-8 \pi V(\varrho+3 p) / 3$ is non-negative for some $V_{+}>V_{\mu}$, then it is non-negative for all $V$ in the interval $\left(V_{\mu}, V_{+}\right]$. 
Proof. Using Eqs. (10) and (11) it is straightforward to evaluate the derivative

$$
\frac{d}{d V}\left(m_{\mu}-\frac{4 \pi}{3} \varrho r_{\mu}^{3}\right)=-\frac{4 \pi}{3} r_{\mu}^{3} \frac{d \varrho}{d V}
$$

The right side of Eq. (25) is non-negative as a consequence of Eq. (4) and the assumed monotonicity of the equation of state. Thus $m_{\mu}-4 \pi \varrho r_{\mu}^{3} / 3$ is non-decreasing on the domain where it is defined: $\left(V_{\mu}, V_{S}\right]$.

Next, we may rewrite Eq. (16) using Eqs. (10) and (11) in the following form:

$$
\frac{d W_{\mu}}{d V}-\frac{8 \pi}{3} V(\varrho+3 p)=-\frac{4 V}{r_{\mu}^{3}}\left(m_{\mu}-\frac{4 \pi}{3} \varrho r_{\mu}^{3}\right) .
$$

The quantity in parenthesis on the right side of Eq. (26) is non-decreasing. If this quantity is non-positive at a point $V_{+}$, then it is non-positive on the interval $\left(V_{\mu}, V_{+}\right]$. Since $r_{\mu}$ and $V$ are positive, it follows that the right side of (26) is non-negative on the interval $\left(V_{\mu}, V_{+}\right]$.

The solutions to Eqs. (10) and (11) in which $W_{\mu}$ vanishes fall into two classes depending on whether $r_{\mu}$ vanishes at $V_{\mu}$ or not. We refer to the case where $\lim _{V \downarrow V_{\mu}} r_{\mu}=0$ as a regular zero of $W_{\mu}$ and the case where $\lim _{V \downarrow V_{\mu}} r_{\mu}>0$ as an irregular zero. The next lemma establishes additional properties of the zeros of $W_{\mu}$.

Lemma 3. Consider a solution to Eqs. (10) and (11) in which $\lim _{V \downarrow V_{\mu}} W_{\mu}=0$ and $\lim _{V \downarrow V_{\mu}} p=p_{\mu}<\infty$. If $\lim _{V \downarrow V_{\mu}} r_{\mu}=0$ (i.e., if $V_{\mu}$ is a regular zero of $W_{\mu}$ ) then $\lim _{V \downarrow V_{\mu}}\left(m_{\mu} / r_{\mu}^{3}\right)=4 \pi \varrho\left(V_{\mu}\right) / 3$ and

$$
\lim _{V \downarrow V_{\mu}} \frac{d W_{\mu}}{d V}=\frac{8 \pi}{3} V_{\mu}\left[\varrho\left(V_{\mu}\right)+3 p_{\mu}\right] .
$$

If $\lim _{V \downarrow V_{\mu}} r_{\mu}>0$ (i.e., if $V_{\mu}$ is an irregular zero) then $\lim _{V \downarrow V_{\mu}}\left(m_{\mu} / r_{\mu}^{3}\right)=-4 \pi p_{\mu}$ and

$$
\lim _{V \downarrow V_{\mu}} \frac{d W_{\mu}}{d V}=8 \pi V_{\mu}\left[\varrho\left(V_{\mu}\right)+3 p_{\mu}\right] .
$$

Proof. For the first case assume that $\lim _{V \downarrow V_{\mu}} r_{\mu}=0$. Using 1'Hospital's rule and Eq. (15) we conclude that $\lim _{V \downarrow V_{\mu}}\left(m_{\mu} / r_{\mu}^{3}\right)=4 \pi \varrho\left(V_{\mu}\right) / 3$. Next, we re-write Eq. (26):

$$
\frac{d W_{\mu}}{d V}-\frac{8 \pi}{3} V(\varrho+3 p)=4 V\left(\frac{4 \pi}{3} \varrho-\frac{m_{\mu}}{r_{\mu}^{3}}\right) .
$$

The right side of Eq. (29) vanishes in the limit $V \downarrow V_{\mu}$, which implies Eq. (27). For the second case assume that $\lim _{V \downarrow V_{\mu}^{\prime}} r_{\mu}>0$. The vanishing of $W_{\mu}$ in the limit $V \downarrow V_{\mu}$ and Eq. (18) imply that $\lim _{V \downarrow V_{\mu}^{\prime}}\left(m_{\mu} / r_{\mu}^{3}\right)=-4 \pi p_{\mu}<0$. This may be used in turn to show that the limit of the right side of Eq. (29) is $16 \pi V_{\mu}\left[\varrho\left(V_{\mu}\right)+3 p_{\mu}\right] / 3$. When combined with the limit of the left side of Eq. (29) this implies Eq. (28). 
We note that the function $W_{\mu}$ defined in Eq. (14) also satisfies the second-order differential equation

$$
\begin{aligned}
\frac{d}{d V} & {\left[\frac{1}{V} \frac{d W_{\mu}}{d V}-8 \pi(\varrho+p)\right] } \\
& =\frac{3}{4 V W_{\mu}}\left[\frac{d W_{\mu}}{d V}-\frac{8 \pi}{3} V(\varrho+3 p)\right]\left[\frac{d W_{\mu}}{d V}-8 \pi V(\varrho+3 p)\right]
\end{aligned}
$$

on the interval $\left(V_{\mu}, V_{S}\right.$ ] where $W_{\mu}>0$. This follows by direct computation using Eqs. (10), (11), and (14).

\section{How $W_{\mu}$ Depends on $\mu$}

In this section we prove several important results about the dependence of $W_{\mu}$ on the parameter $\mu$. First we show that $W_{\mu}$ is a $C^{1}$ function of $\mu$ on the domain where it is well defined

Lemma 4. The functions $r_{\mu}(V), m_{\mu}(V)$, and $W_{\mu}(V)$, as defined in Eqs. (10)-(14), are $C^{1}$ with respect to variations in $\mu$ (at fixed $V$ ) for $\mu>0$ and for $V$ in the interval $\left(V_{\mu}, V_{S}\right]$.

Proof. It is useful to consider the functions $r_{\mu}(V), m_{\mu}(V)$, and $W_{\mu}(V)$ as the components of a three-dimensional vector:

$$
\vec{Y}_{\mu}(V)=\left[\begin{array}{c}
r_{\mu}(V) \\
m_{\mu}(V) \\
W_{\mu}(V)
\end{array}\right]
$$

This vector satisfies the ordinary differential equation

$$
\frac{d \vec{Y}_{\mu}}{d V}=\vec{F}\left(\vec{Y}_{\mu}, V\right),
$$

when $\vec{F}\left(\vec{Y}_{\mu}, V\right)$ is given by

$$
\vec{F}\left(\vec{Y}_{\mu}, V\right)=\left[\begin{array}{c}
\left(1-2 m_{\mu} / r_{\mu}\right)^{1 / 2} W_{\mu}^{-1 / 2} \\
4 \pi \varrho r_{\mu}^{2}\left(1-2 m_{\mu} / r_{\mu}\right)^{1 / 2} W_{\mu}^{-1 / 2} \\
8 \pi V(\varrho+p)-4 V m_{\mu} r_{\mu}^{-3}
\end{array}\right]
$$

Equation (32) is equivalent to Eqs. (10), (11), and (16). We note that $W_{\mu}>0, r_{\mu}>0$, and $r_{\mu}>2 m_{\mu}$ on the interval $\left(V_{\mu}, V_{S}\right]$ as a consequence of Lemma 1 . The equation of state $\varrho(p)$ was assumed to be a $C^{1}$ function for $p>0$, which implies that $p(V)$ and $\varrho(V)$ are also $C^{1}$ for $V<V_{S}$. These functions, $\varrho(V)$ and $p(V)$, can also be extended as $C^{0}$ functions for $V \geq V_{S}$. Consequently, $\vec{F}\left(\vec{Y}_{\mu}, V\right)$ is a $C^{1}$ function of $\vec{Y}$ and a $C^{0}$ function of $V$, for $V$ in an open interval containing $\left(V_{\mu}, V_{S}\right]$. Standard theorems on systems of ordinary differential equations (see e.g., [16, Theorem 7.2, p. 25]) guarantee then that the solutions of Eq. (32) are $C^{1}$ functions of the initial 
conditions $\vec{Y}_{\mu}\left(V_{S}\right)$. (We point out that these theorems do not require $\varrho(V)$ and $p(V)$ to be differentiable at $V=V_{S}$.) The initial conditions of particular interest to us,

$$
\vec{Y}_{\mu}\left(V_{S}\right)=\left[\begin{array}{c}
2 \mu /\left(1-V_{S}^{2}\right) \\
\mu \\
\left(1-V_{S}^{2}\right)^{4} / 16 \mu^{2}
\end{array}\right],
$$

are $C^{1}$ functions of $\mu$ when $\mu>0$. It follows that $r_{\mu}(V), m_{\mu}(V)$, and $W_{\mu}(V)$ are $C^{1}$ functions of $\mu$ for $V$ in the interval $\left(V_{\mu}, V_{S}\right.$ ] and for $\mu>0$. This continuity condition on these functions may be summarized as follows: There exists a $\bar{\delta}>0$ such that for every $\delta \in(-\bar{\delta}, \bar{\delta})$ the functions $\vec{Y}_{\mu}$ and their derivatives satisfy

$$
\left|\vec{Y}_{\mu}(V)-\vec{Y}_{\mu+\delta}(V)\right|+\left|\frac{d \vec{Y}_{\mu}}{d V}(V)-\frac{d \vec{Y}_{\mu+\delta}}{d V}(V)\right| \leq C \delta,
$$

where $C=C(V, \mu)$ is independent of $\delta$.

The next lemma demonstrates that $W_{\mu}$ is monotonic in addition to being differentiable with respect to the parameter $\mu$. Our proof uses the fact that the function $\Sigma_{\nu}$, defined by

$$
\Sigma_{\nu}=\frac{d W_{\nu}}{d V}-\frac{8 \pi}{3} V(\varrho+3 p)+\frac{4 W_{\nu}}{5 V} \frac{\varrho+p}{\varrho+3 p} \frac{d \varrho}{d p},
$$

is non-negative. We delay until Sect. 4 the proof that $\Sigma_{\nu} \geq 0$ and merely state this condition here as an assumption.

Lemma 5. Assume that $\Sigma_{\nu}(V) \geq 0$ for $V$ in the interval $\left(V_{\nu}, V_{S}\right)$. If $\mu>\nu$ then $W_{\mu}(V)<W_{\nu}(V)$ on $\left(V_{\mu}, V_{S}\right]$; and if $\mu<\nu$ then $W_{\mu}(V)>W_{\nu}(V)$ on $\left(V_{\nu}, V_{S}\right]$.

Proof To facilitate comparison between $W_{\mu}$ and $W_{\nu}$ we introduce the function

$$
\Delta_{\mu \nu}=\frac{W_{\mu}-W_{\nu}}{\lambda_{\nu}^{4}},
$$

for $V$ in the interval $\left(V_{\mu}, V_{S}\right] \cap\left(V_{\nu}, V_{S}\right.$. The function $\lambda_{\nu}$ is defined by

$$
\lambda_{\nu}(V)=W_{\nu}(V)^{1 / 4} \frac{2 \nu^{1 / 2}}{1-V_{S}^{2}} \exp \left\{\frac{2 \pi}{3} \int_{V}^{V_{S}} \frac{\widehat{V}[\varrho(\widehat{V})+3 p(\widehat{V})]}{W_{\nu}(\widehat{V})} d \widehat{V}\right\} .
$$

From Eq. (38) it follows that $\lambda_{\nu}(V)>0$, and that $\lambda_{\nu}$ satisfies the differential equation

$$
\frac{d \lambda_{\nu}}{d V}=\frac{\lambda_{\nu}}{4 W_{\nu}}\left[\frac{d W_{\nu}}{d V}-\frac{8 \pi}{3} V(\varrho+3 p)\right]
$$

with the boundary condition $\lambda_{\nu}\left(V_{S}\right)=1$. A lengthy but straightforward calculation using Eqs. (30), (36), and (39) shows that $\Delta_{\mu \nu}$ satisfies the differential equation

$$
\begin{aligned}
\frac{d^{2} \Delta_{\mu \nu}}{d V^{2}}= & \frac{20 \pi V}{3 W_{\nu}^{2}}(\varrho+3 p) \Sigma_{\nu} \Delta_{\mu \nu} \\
& +\left[\frac{1}{V}-\frac{2}{\lambda_{\nu}} \frac{d \lambda_{\nu}}{d V}-\frac{4 \pi V}{W_{\mu}}(\varrho+3 p)+\frac{3 \lambda_{\nu}^{4}}{4 W_{\mu}} \frac{d \Delta_{\mu \nu}}{d V}\right] \frac{d \Delta_{\mu \nu}}{d V}
\end{aligned}
$$


for $V$ in the interval $\left(V_{\mu}, V_{S}\right) \cap\left(V_{\nu}, V_{S}\right)$. Equation (40) is a degenerate special case of an identity given in Beig and Simon [6,7]. The particular solution of (40) that is of interest to us, Eq. (37), satisfies the boundary conditions

$$
\begin{aligned}
\lim _{V \uparrow V_{S}} \Delta_{\mu \nu} & =\frac{\left(1-V_{S}\right)^{4}}{16}\left(\frac{1}{\mu^{2}}-\frac{1}{\nu^{2}}\right), \\
\lim _{V \uparrow V_{S}} \frac{d \Delta_{\mu \nu}}{d V} & =\frac{16 \pi}{3} V_{S} \varrho\left(V_{S}\right)\left(1-\frac{\nu^{2}}{\mu^{2}}\right),
\end{aligned}
$$

as a consequence of Eqs. (37), (12)-(14), (16), and (38).

The assumption $\Sigma_{\nu} \geq 0$ guarantees that the coefficient of $\Delta_{\mu \nu}$ on the right side of Eq. (40) is non-negative. Therefore the solutions of Eq. (40) satisfy a maximum principle: $\Delta_{\mu \nu}$ may have no negative minimum or positive maximum except at the endpoints of the interval $\left(V_{\mu}, V_{S}\right) \cap\left(V_{\nu}, V_{S}\right)$, unless it is constant.

Assume first that $\mu>\nu$. It follows from Eqs. (41) and (42) that $\Delta_{\mu \nu}\left(V_{S}\right)<0$ and that $d \Delta_{\mu \nu}\left(V_{S}\right) / d V \geq 0$. If $\varrho\left(V_{S}\right)>0$ then $d \Delta_{\mu \nu}\left(V_{S}\right) / d V>0$ so that $\Delta_{\mu \nu}$ is strictly increasing at $V=V_{S}$. Thus $\Delta_{\mu \nu}$ must be negative on the entire interval $\left(V_{\mu}, V_{S}\right) \cap\left(V_{\nu}, V_{S}\right)$ unless it has a minimum where its value is negative. Such a minimum is excluded, however, by the maximum principle. The case $\varrho\left(V_{S}\right)=0$ is more delicate and must be analyzed with the more powerful boundary point maximum principle: if a negative minimum of $\Delta_{\mu \nu}$ is reached at $V_{S}$ then $d \Delta_{\mu \nu} / d V$ must be negative there, or $\Delta_{\mu \nu}$ must be constant. But this contradicts Eq. (42) and so the minimum of $\Delta_{\mu \nu}$ cannot be reached at $V_{S}$ unless it is a constant everywhere. Thus, $\Delta_{\mu \nu}$ must be negative everywhere on $\left(V_{\mu}, V_{S}\right) \cap\left(V_{\nu}, V_{S}\right)$ because the possibility of a negative minimum elsewhere is excluded by the regular maximum principle. We conclude that $\Delta_{\mu \nu}<0$ everywhere on the interval $\left(V_{\mu}, V_{S}\right) \cap\left(V_{\nu}, V_{S}\right)$ for the case $\mu>\nu$. Therefore $W_{\mu}<W_{\nu}$ on the interval $\left(V_{\mu}, V_{S}\right.$ ]. The proof that $W_{\mu}>W_{\nu}$ on the interval $\left(V_{\nu}, V_{S}\right]$ for the case $\mu<\nu$ is exactly analogous.

Lemma 1 shows that the solutions to Eqs. (10) and (11) exist up to the point $V=V_{\mu}$, where $W_{\mu}$ vanishes or the pressure diverges, and the last lemma shows how the $W_{\mu}$ depend on $\mu$. An important element of our subsequent analysis will be a knowledge of the relationship between the functions $W_{\mu}(V)$ and $W$ as defined in Eq. (7). The following lemma derives one important relationship.

Lemma 6. Let $V_{C}>0$ denote the minimum value of $V$ in the spacetime of a static stellar model of mass $M$. Assume that $W_{\mu}>0$ and $\Sigma_{\mu} \geq 0$ on $\left(V_{C}, V_{S}\right]$. Then $W_{\mu} \geq W$ on $\left(V_{C}, V_{S}\right]$ and $\mu \leq M$.

Proof. The difference between $W$ [as defined in Eq. (7)] and $W_{\mu}$ is conveniently characterized by the function,

$$
\Delta_{\mu}^{-} \equiv \frac{W-W_{\mu}}{\lambda_{\mu}^{4}},
$$

where $\lambda_{\mu}$ is defined in Eq. (38). Beig and Simon [6,7] have shown that $\Delta_{\mu}^{-}$satisfies the identity,

$$
\begin{gathered}
D_{a}\left(\frac{\lambda_{\mu}^{2}}{V} D^{a} \Delta_{\mu}^{-}\right)-\frac{20 \pi}{3} \frac{W}{W_{\mu}^{2}}(\varrho+3 p) \lambda_{\mu}^{2} \Sigma_{\mu} \Delta_{\mu}^{-} \\
\quad=\frac{V^{3}}{4 \lambda_{\mu}^{2} W} R^{a b c} R_{a b c}+\frac{3 \lambda_{\mu}^{6}}{4 V W} D_{a} \Delta_{\mu}^{-} D^{a} \Delta_{\mu}^{-}
\end{gathered}
$$


on the domain $V_{C}<V \leq V_{S}$. The tensor $R_{a b c}$ is defined by

$$
R_{a b c}=D_{c} R_{a b}-D_{b} R_{a c}+\frac{1}{4}\left(g_{a c} D_{b} R-g_{a b} D_{c} R\right) \text {. }
$$

Equation (44) is an elliptic equation for $\Delta_{\mu}^{-}$whose right side is non-negative. Since $\Sigma_{\mu}>0$ the maximum principle implies that any positive maximum of $\Delta_{\mu}^{-}$must occur on the boundary of this domain, and the outward directed normal derivative must be positive at this maximum point, $n^{a} D_{a} \Delta_{\mu}^{-}>0$, unless $\Delta_{\mu}^{-}$is constant. We note that if the maximum of $\Delta_{\mu}^{-}$occurs at $V_{C}$ or some other critical point of $W$ then $\Delta_{\mu}^{-} \leq 0$ automatically since $W=0$ at any critical point. We also note that when $\Delta_{\mu}^{-}$ is strictly positive at the maximum point, we can use the boundary point maximum principle (see e.g., [17, Lemma 3.4]) since Eq. (44) implies $D_{a}\left(V^{-1} \lambda_{\mu}^{2} D^{a} \Delta_{\mu}^{-}\right) \geq 0$ in this case. It follows that if $\Delta_{\mu}^{-}>0$ at its maximum point which occurs at the surface $V=V_{S}$, then

$$
n^{a} D_{a} \Delta_{\mu}^{-}=n^{a} D_{a}\left(W-W_{\mu}\right)-4\left(W-W_{\mu}\right) \frac{d \lambda_{\mu}}{d V} n^{a} D_{a} V>\varepsilon \Delta_{\mu}^{-} n^{a} D_{a} V,
$$

at the maximum for some $\varepsilon>0$.

In the exterior of the stellar model, $V_{S}<V<1$, we define

$$
W_{\mu}=\frac{\left(1-V^{2}\right)^{4}}{16 \mu^{2}} \text {. }
$$

This extension of $W_{\mu}$ is $C^{0}$ across the surface $V=V_{S}$, and the quantity $W-W_{\mu}$ is $C^{1}$ there. As Eq. (8) illustrates, however, neither $W$ nor $W_{\mu}$ will be $C^{1}$ at $V=V_{S}$ if $\varrho\left(V_{S}\right) \neq 0$. Near spatial infinity the asymptotic behavior of $W_{\mu}$ can be deduced from its definition and Eq. (5) to be

$$
W_{\mu}=\frac{M^{4}}{\mu^{2} r^{4}}+O\left(r^{-5}\right) .
$$

For comparison, the asymptotic form for $W$ is

$$
W=\frac{M^{2}}{r^{4}}+O\left(r^{-5}\right)
$$

We compare $\dot{W}_{\mu}$ to $W$ in the exterior of the star by making use of the quantity

$$
\Delta_{\mu}^{+}=\left(W-W_{\mu}\right) \frac{\left(1-V_{S}^{2}\right)^{3}\left(1+b-V_{S}^{2}\right)}{\left(1-V^{2}\right)^{3}\left(1+b-V^{2}\right)},
$$

where $b$ is a positive constant to be determined later. Robinson [18] showed that $\Delta_{\mu}^{+}$ satisfies the identity

$$
D_{a}\left(V^{-1} D^{a} \Delta_{\mu}^{+}\right)=\left(V^{4} R_{a b c} R^{a b c}+3 X^{a} X_{a}\right) \frac{\left(1-V_{S}^{2}\right)^{3}\left(1+b-V_{S}^{2}\right)}{4 V W\left(1-V^{2}\right)^{3}\left(1+b-V^{2}\right)},
$$

in the exterior vacuum region for any value of $b$. The quantity $X_{a}$ is defined by $X_{a}=D_{a} W+8 V W\left(1-V^{2}\right)^{-1} D_{a} V$. Since the right side of Eq. (51) is non-negative, the maximum principle implies that $\Delta_{\mu}^{+}$may have a maximum only at infinity (where it vanishes) or on the surface of the star $V=V_{S}$. If the maximum of $\Delta_{\mu}^{+}$occurs at infinity, then $W-W_{\mu} \leq 0$ everywhere. The alternative is that $\Delta_{\mu}^{+}$has a positive 
maximum on the surface $V=V_{S}$. In this case the boundary maximum principle implies that the outward directed normal derivative is negative there:

$$
n^{a} D_{a} \Delta_{\mu}^{+}<0 \text {. }
$$

But this condition contradicts Eq. (46) for appropriately chosen values of $b$. If

$$
\widehat{\varepsilon} \equiv \varepsilon+\frac{256 \pi}{3} \frac{\mu^{2} V_{S} \varrho\left(V_{S}\right)}{\left(1-V_{S}^{2}\right)^{4}} \geq \frac{2 V_{S}}{1-V_{S}^{2}},
$$

then Eq. (52) is inconsistent with (46) for any choice of $b>0$. However if $\widehat{\varepsilon}$ violates the inequality Eq. (53) then the choice

$$
0<b<\frac{\widehat{\varepsilon}\left(1-V_{S}^{2}\right)^{2}}{2 V_{S}-\widehat{\varepsilon}\left(1-V_{S}^{2}\right)},
$$

makes Eq. (52) inconsistent with (46). Thus, $\Delta_{\mu}^{+}$may have no positive maximum on the surface $V=V_{S}$. Thus, we have established the first conclusion of the lemma: $W_{\mu} \geq W$ everywhere. The second conclusion, $\mu \leq M$, follows by imposing the inequality $W_{\mu} \geq W$ in a neighborhood of spatial infinity. The asymptotic expansions in Eqs. (48) and (49) imply then that $\mu \leq M$.

Next we prove a result which, when combined with the assumptions about the equation of state to be made in Sect. 4, demonstrates that there exists a value of the mass parameter $\mu$ for which $W_{\mu}$ has a zero inside the star, i.e., in the interval $\left(V_{C}, V_{S}\right]$.

Lemma 7. There exists a $\mu>M$ such that $V_{\mu} \in\left(V_{C}, V_{S}\right]$ or $\Sigma_{\mu} \geq 0$ on the interval $\left[V_{C}, V_{+}\right]$for some $V_{+} \in\left(V_{C}, V_{S}\right]$.

Proof. Consider first the case where $\varrho\left(V_{S}\right)>0$. Then we choose a $\mu>M$ such that,

$$
\mu>\sqrt{\frac{3\left(1-V_{S}^{2}\right)^{3}}{32 \pi \varrho\left(V_{S}\right)}} .
$$

For this choice it follows that

$$
\mu-4 \pi \varrho\left(V_{S}\right) R_{\mu}^{3} / 3<0 .
$$

From Lemma 2, then $m_{\mu}-4 \pi \varrho r_{\mu}^{3} / 3<0$ on $\left(V_{\mu}, V_{S}\right]$. It follows then from Eq. (26) and the definition of $\Sigma_{\mu}$, Eq. (36), that $\Sigma_{\mu} \geq 0$ on $\left(V_{\mu}, V_{S}\right]$ as well. If $V_{\mu} \notin\left(V_{C}, V_{S}\right]$ then $W_{\mu}>0$ and $\Sigma_{\mu} \geq 0$ on $\left(V_{C}, V_{S}\right]$. But this contradicts Lemma 6 since we assumed $\mu>M$. Thus, we conclude that $V_{\mu} \in\left(V_{C}, V_{S}\right]$.

Consider next the case where $\varrho\left(V_{S}\right)=0$. Let $\delta \in\left(0,10^{-3}\right)$ and then choose $V_{*} \in\left(V_{C}, V_{S}\right)$ such that

$$
\log \left(\frac{V_{S}}{V_{*}}\right)<\frac{\delta}{4}\left(1-V_{S}^{2}\right) .
$$

Next choose a $\mu>M$ such that

$$
\mu>\sqrt{\frac{3\left(1-V_{S}^{2}\right)^{3}}{16 \pi \varrho\left(V_{*}\right)}},
$$

so that

$$
\mu<2 \pi R_{\mu}^{3} \varrho\left(V_{*}\right) / 3
$$


If $V_{\mu} \in\left(V_{C}, V_{S}\right.$ ] the proof would be complete; thus we consider only the case $V_{\mu} \notin\left(V_{C}, V_{S}\right]$. It follows from Lemma 1 that $r_{\mu}$ and $m_{\mu}$ are defined on $\left[V_{C}, V_{\mu}\right]$ in this case. Near the surface of the star we must have

$$
\frac{m_{\mu}}{r_{\mu}^{3}}-\frac{4 \pi}{3} \varrho>0
$$

since $\varrho\left(V_{S}\right)=0$. If the left side of Eq. (60) vanishes at any point $V_{+}$within the star, then it follows from Lemma 2, Eq. (26), and the definition of $\Sigma_{\mu}$, Eq. (36), that $\Sigma_{\mu} \geq 0$ on $\left[V_{C}, V_{+}\right]$as claimed. We will now show that the left side of Eq. (60) always vanishes somewhere. Thus, we suppose to the contrary that Eq. (60) is satisfied on the entire interval $\left[V_{C}, V_{S}\right]$. Equation (15) may now be used to obtain the following expression,

$$
\frac{d}{d r_{\mu}}\left(\frac{m_{\mu}}{r_{\mu}^{3}}\right)=\frac{1}{r_{\mu}}\left(4 \pi \varrho-\frac{3 m_{\mu}}{r_{\mu}^{3}}\right) .
$$

For our case the right side of Eq. (61) is negative and so $m_{\mu} / r_{\mu}^{3}$ is strictly decreasing as $r_{\mu}$ increases. Integrating Eq. (61) then we obtain a useful estimate:

$$
\frac{\mu}{R_{\mu}^{3}}-\frac{m_{\mu}}{r_{\mu}^{3}} \geq-\int_{r_{\mu}}^{R_{\mu}} \frac{3 \widehat{m}_{\mu}}{\widehat{r}_{\mu}^{4}} d \widehat{r}_{\mu} \geq-\frac{m_{\mu}}{r_{\mu}^{3}} \int_{r_{\mu}}^{R_{\mu}} \frac{3 d \widehat{r}_{\mu}}{\widehat{r}_{\mu}}=-\frac{3 m_{\mu}}{r_{\mu}^{3}} \log \left(\frac{R_{\mu}}{r_{\mu}}\right) .
$$

This implies that

$$
\frac{\mu}{R_{\mu}^{3}} \geq \frac{m_{\mu}}{r_{\mu}^{3}}\left[1-3 \log \left(\frac{R_{\mu}}{r_{\mu}}\right)\right] .
$$

Now since $m_{\mu} / r_{\mu}^{3}$ is strictly decreasing as $r_{\mu}$ increases we may also obtain from Eq. (10) the inequality

$$
\frac{d r_{\mu}^{2}}{d V}=\frac{2 r_{\mu}^{2}\left(r_{\mu}-2 m_{\mu}\right)}{V\left(m_{\mu}+4 \pi r_{\mu}^{3} p\right)} \leq \frac{2 r_{\mu}^{3}}{V m_{\mu}} \leq \frac{2 R_{\mu}^{3}}{V_{\mu}}
$$

on $\left[V_{C}, V_{S}\right]$. Integrating this expression we get

$$
\frac{r_{\mu}^{2}}{R_{\mu}^{2}} \geq 1-\frac{4}{1-V_{S}^{2}} \log \left(\frac{V_{S}}{V}\right)
$$

and so

$$
r_{\mu}^{2}(V) \geq r_{\mu}^{2}\left(V_{*}\right) \geq(1-\delta) R_{\mu}^{2},
$$

for $V \in\left[V_{*}, V_{S}\right.$ ). Now using Eqs. (63) and (66) we get

$$
\frac{\mu}{R_{\mu}^{3}} \geq \frac{m_{\mu}}{r_{\mu}^{3}}\left[1+\frac{3}{2} \log (1-\delta)\right]>0.9 \frac{m_{\mu}}{r_{\mu}^{3}},
$$

for $\delta<10^{-3}$. Using Eqs. (59) and (67) we find that

$$
\frac{m_{\mu}}{r_{\mu}^{3}}-\frac{4 \pi}{3} \varrho<\frac{m_{\mu}}{0.9 R_{\mu}^{3}}-\frac{4 \pi}{3} \varrho \leq \frac{2 \pi}{3}\left[\frac{\varrho\left(V_{*}\right)}{0.9}-2 \varrho(V)\right],
$$

on $\left[V_{*}, V_{S}\right)$. But the right-hand side is negative at $V=V_{*}$ which contradicts our assumption that Eq. (60) is satisfied everywhere. 
Complementary to the previous results, we now show that if the mass parameter $\mu$ is sufficiently small, then the function $W_{\mu}$ exceeds any given constant. In particular this lemma shows that $W_{\mu}>\sup W \geq W$ on $\left[V_{C}, V_{S}\right.$ ] if $\mu$ is sufficiently small.

Lemma 8. Let $K>0$ be a constant and $\left(V_{m}, V_{S}\right.$ ] an interval on which $p(V)$ is bounded. Then there exists a constant $\mu_{K}>0$ such that $W_{\mu}>K$ on $\left(V_{m}, V_{S}\right]$ for all $\mu \in\left(0, \mu_{K}\right)$.

Proof. Define $\mu_{K}$ by the condition

$$
\frac{1}{\mu_{K}^{2}}=\frac{16}{\left(1-V_{S}^{2}\right)^{4}}\left[K+8 \pi \int_{V_{m}}^{V_{S}} \widehat{V}(\widehat{\varrho}+3 \widehat{p}) d \widehat{V}\right] .
$$

Next we rewrite Eq. (17) in the form

$$
W_{\mu}=\left(\frac{R_{\mu}}{r_{\mu}}\right)^{4}\left[\frac{\left(1-V_{S}^{2}\right)^{4}}{16 \mu^{2}}-8 \pi \int_{V}^{V_{S}} \widehat{V}(\widehat{\varrho}+3 \widehat{p})\left(\frac{\widehat{r}_{\mu}}{R_{\mu}}\right)^{4} d \widehat{V}\right] .
$$

From Lemma 1 we know that $0<r_{\mu} \leq R_{\mu}$ on the domain $\left(V_{\mu}, V_{S}\right.$, here $W_{\mu}>0$ and $p(V)$ is bounded. Thus, the integral on the right side of Eq. (70) can be estimated to yield a lower bound on $W_{\mu}$ for points in the interval $\left(V_{\mu}, V_{S}\right] \cap\left(V_{m}, V_{S}\right]$ :

$$
W_{\mu}(V) \geq\left(\frac{R_{\mu}}{r_{\mu}}\right)^{4}\left[\frac{\left(1-V_{S}^{2}\right)^{4}}{16 \mu^{2}}-8 \pi \int_{V_{m}}^{V_{S}} \widehat{V}(\widehat{\varrho}+3 \widehat{p}) d \widehat{V}\right] \geq K
$$

The second inequality in Eq. (71) is satisfied for $\mu<\mu_{K}$. Since $W_{\mu}>K>0$ and $p(V)$ is bounded on the interval $\left(V_{\mu}, V_{S}\right] \cap\left(V_{m}, V_{S}\right]$ we know from Lemma 1 that this must be a subset of $\left(V_{\mu}, V_{S}\right.$ ]. Thus $W_{\mu}>K$ on $\left(V_{m}, V_{S}\right]$ for $\mu<\mu_{K}$.

\section{Conditions on the Equation of State}

Up to this point we have assumed only that the equation of state $\varrho=\varrho(p)$ is a positive, non-decreasing, $C^{1}$ function for $p>0$. We know that some additional conditions on the equation of state are also implicitly required to have existence of static stellar models with finite mass and radius, see e.g., [8, 19-21]. We presume that spherical symmetry is a necessary feature of any static stellar model whose equation of state satisfies the minimal monotonicity and smoothness assumptions that we have used up to now, plus whatever additional minimal conditions on the equation of state are required to guarantee the existence of static stellar models. At the present time, however, additional unphysical restrictions on the equation of state must be used in order to complete a proof of the necessity of spherical symmetry. An additional restriction on the equation of state is needed, in particular, to prove that the functions $\Sigma_{\mu}$, defined in Eq. (36), are positive; and a different condition is needed to prove that the conformal factor $\psi_{\mu}(V)$, defined in Sect. 6 below, has non-negative second derivative. Two alternate conditions on the equation of state have been found which allow us to prove these results. The first restriction (introduced by Beig and Simon 
$[6,7])$ assumes that the equation of state is a $C^{2}$ function whose second derivative satisfies the condition,

$$
\frac{1}{5} \kappa^{2}+2 \kappa+(\varrho+p) \frac{d \kappa}{d p} \equiv I \leq 0
$$

where $\kappa$ is defined by

$$
\kappa \equiv \frac{\varrho+p}{\varrho+3 p} \frac{d \varrho}{d p} .
$$

We note that the condition $I \leq 0$ implies an upper bound on the value of $\kappa$. In particular, Eq. (72) can be integrated,

$$
\begin{aligned}
\log \left\{\frac{\kappa(p)[10+\kappa(0)]}{\kappa(0)[10+\kappa(p)]}\right\} & =10 \int_{0}^{p} \frac{d \kappa}{d p^{\prime}} \frac{d p^{\prime}}{\kappa\left(p^{\prime}\right)\left[\kappa\left(p^{\prime}\right)+10\right]} \\
& \leq-2 \int_{0}^{p} \frac{d p^{\prime}}{\varrho\left(p^{\prime}\right)+p^{\prime}}=-2 h(p)
\end{aligned}
$$

where $\kappa(0) \equiv \lim _{p \downarrow 0} \kappa(p)$. This inequality is equivalent to the bound,

$$
\kappa(p) \leq \frac{10 \kappa(0)}{[\kappa(0)+10] \exp [2 h(p)]-\kappa(0)} \leq \frac{10}{\exp [2 h(p)]-1} .
$$

Our alternate condition on the equation of state places upper and lower limits on the value of $\kappa$ for stars with a given value of $V_{S}$,

$$
\frac{5 \varrho^{2}}{6 p(\varrho+3 p)} \geq \kappa>\frac{10 V_{S}^{2}}{\exp [2 h(p)]-V_{S}^{2}} \equiv \kappa_{0}(p)
$$

but no limit on its derivative. The two conditions on the equation of state, Eq. (72) (Assumption A) and Eq. (76) (Assumption B) are complementary: Assumption A limits the second derivative of the equation of state, while Assumption $\mathbf{B}$ does not. Assumption $\mathbf{B}$ places a positive lower bound on $\kappa$, while assumption $\mathbf{A}$ does not. We also point out that conditions $\mathbf{A}$ and $\mathbf{B}$ are in effect constraints on the adiabatic index, $\gamma=(\varrho+p)(d \log p / d \varrho)$, of the fluid and its first derivative.

Before proceeding it is appropriate to point out that each set of equations of state defined by conditions A or $\mathbf{B}$ is not empty. It is easy to verify, for example, that the one parameter family of equations of state

$$
\kappa(p)=\frac{10 \kappa(0)}{[\kappa(0)+10] \exp [2 h(p)]-\kappa(0)},
$$

satisfies Condition A. In fact $I=0$ for this family. The parameter $\kappa(0)$ is limited only by the requirement that it be positive in order to insure that $\varrho(p)$ (obtained by integrating Eqs. (9) and (73)) is an increasing function. It is also easy to verify (using Lemma 9 below) that the equations of state in Eq. (77) satisfy Condition $\mathbf{B}$ if $\kappa(0)$ is restricted to the range $\kappa(0)>10 V_{S}^{2} /\left(1-V_{S}^{2}\right)$. We note that $\mathbf{B}$ is a restriction on the space of equations of state for stars of given $V_{S}$. Our principle result in this paper eliminates the possibility of non-spherical stars with equations of state that satisfy one of these restrictions. For some values of $V_{S}$ (e.g., for $V_{S}<1 / 3$, see Buchdahl 
[22]) there exists no spherical stellar model whose equation of state satisfies these conditions.

The next lemma shows that Assumption A implies the first of the inequalities in Assumption B:

Lemma 9. Assume that the equation of state $\varrho=\varrho(p)$ is a non-negative, nondecreasing, $C^{2}$ function which satisfies the inequality $I \leq 0$ defined in $E q$. (72). Then $5 \varrho^{2} \geq 6 p(\varrho+3 p) \kappa$.

Proof. The proof follows exactly the proof of Lemma 5 of Beig and Simon [7].

We next prove that either of these assumptions is sufficient to guarantee that the quantity $\Sigma_{\mu}$ defined in Eq. (36) is positive.

Lemma 10. If the equation of state satisfies Condition $\mathbf{A}, E q$ (72), and $W_{\mu}$ satisfies $\lim _{V \downarrow V_{\mu}} W_{\mu}=0$, where $\lim _{V \downarrow V_{\mu}} p=p_{\mu}<\infty$, then the function $\Sigma_{\mu}$ defined in Eq. (36) is non-negative on the interval $\left(V_{\mu}, V_{S}\right]$. If the equation of state satisfies Condition $\mathbf{A}$ and if $\Sigma_{\mu} \geq 0$ at some $V_{+}$such that $W_{\mu}>0$ on $\left[V_{+}, V_{S}\right]$, then $\Sigma_{\mu}>0$ on $\left[V_{+}, V_{S}\right]$. And finally, if the equation of state satisfies Condition $\mathbf{B}, E q(76)$, then $\Sigma_{\mu} \geq 0$ on $\left(V_{\mu}, V_{S}\right]$.

Proof. Assume first that the equation of state satisfies Condition A: $I \leq 0$. The proof in this case follows almost exactly the proof of Lemma 7 of Beig and Simon [7]. First define the quantity,

$$
t_{\mu}=\frac{\Sigma_{\mu}}{2 V W_{\mu}^{1 / 2}}
$$

which satisfies the differential equation

$$
\frac{d t_{\mu}}{d V}=\frac{V t_{\mu}^{2}}{2 W_{\mu}^{1 / 2}}-\frac{16 \pi V}{3 W_{\mu}}(\varrho+3 p) t_{\mu}-\frac{2 W_{\mu}^{1 / 2} I}{5 V^{3}} .
$$

In the case where $V_{\mu}$ is a regular zero, Beig and Simon [7] have shown that $\lim _{V \downarrow V_{\mu}} t_{\mu}=0$, while for an irregular zero it is easy to see that $t_{\mu}>0$ for $V$ slightly larger than $V_{\mu}$ (although it diverges at $V=V_{\mu}$ ). If $t_{\mu}$ were negative, then $d t_{\mu} / d V>0$ as a consequence of Eq. (79). But since $t_{\mu} \geq 0$ at $V=V_{\mu}$ it follows that $t_{\mu}$ (and hence $\left.\Sigma_{\mu}\right)$ must remain non-negative on all of $\left(V_{\mu}, V_{S}\right)$.

Under the second condition $\Sigma_{\mu} \geq 0$, and hence $t_{\mu} \geq 0$ at $V=V_{+}$. The same argument used in the first case implies then that $t_{\mu}$ must remain non-negative for larger values of $V$. Thus, $t_{\mu} \geq 0$ and hence $\Sigma_{\mu} \geq 0$ on $\left[V_{+}, V_{S}\right]$.

Consider finally the case when the equation of state satisfies Condition B, Eq. (76). We define the function $\widehat{\Sigma}_{\mu}$,

$$
\widehat{\Sigma}_{\mu}=\frac{d W_{\mu}}{d V}-\frac{8 \pi}{3} V(\varrho+3 p)+\frac{4 W_{\mu}}{5 V} \kappa_{0}(p)
$$

which satisfies the inequality

$$
\Sigma_{\mu}-\widehat{\Sigma}_{\mu}=\frac{4 W_{\mu}}{5 V}\left(\kappa-\kappa_{0}\right)>0,
$$


when $\kappa_{0}$ is defined as in Eq. (76). Thus, $\widehat{\Sigma}_{\mu}$ is a lower bound for $\Sigma_{\mu}$. The limiting value of $\widehat{\Sigma}_{\mu}$ at the surface $V=V_{S}$ may be determined with the aid of Eq. (29),

$$
\lim _{V \uparrow V_{S}} \widehat{\Sigma}_{\mu}=\frac{16 \pi}{3} V_{S} \varrho\left(V_{S}\right)+\frac{\left(1-V_{S}^{2}\right)^{4}}{20 V_{S} \mu^{2}}\left[\kappa_{0}(0)-\frac{10 V_{S}^{2}}{1-V_{S}^{2}}\right] .
$$

Since $h(0)=0$, it follows that the term in square brackets on the right side of Eq. (82) vanishes. Thus, $\lim _{V \uparrow V_{S}} \widehat{\Sigma}_{\mu} \geq 0$. Next, we evaluate the derivative of $\widehat{\Sigma}_{\mu}$ using Eq. (30) and (76). It follows that $\widehat{\Sigma}_{\mu}$ satisfies the differential equation

$$
\frac{d \widehat{\Sigma}_{\mu}}{d V}-\left[\frac{1}{V}+\frac{3 \widehat{\Sigma}_{\mu}}{4 W_{\mu}}-\frac{2 \kappa_{0}}{5 V}-\frac{4 \pi V}{W_{\mu}}(\varrho+3 p)\right] \widehat{\Sigma}_{\mu}=-\frac{16 \pi}{3}(\varrho+3 p)\left(\kappa-\kappa_{0}\right) .
$$

The right side of Eq. (83) is negative, therefore the derivative $d \widehat{\Sigma}_{\mu} / d V$ is negative at any point where $\widehat{\Sigma}_{\mu}$ vanishes. Since $\widehat{\Sigma}_{\mu} \geq 0$ at $V=V_{S}$ from Eq. (82), it follows that $\widehat{\Sigma}_{\mu} \geq 0$ for all $V \leq V_{S}$. We conclude that $\Sigma_{\mu}>0$ from Eq. (81).

We point out that Lemma 10 strengthens the conclusion of Lemma 7. In particular when either condition on the equation of state is assumed then Lemma 7 implies that there exists a $\mu>M$ such that $V_{\mu} \in\left[V_{C}, V_{S}\right]$ or that $\Sigma_{\mu} \geq 0$ on $\left[V_{C}, V_{S}\right]$.

\section{Choosing the Reference Spherical Model}

In this section we prove several results whose purpose is to identify the appropriate value of the mass parameter $\mu$ to choose for the "reference spherical model." Let $M^{+}>M$ denote a value of the mass parameter such that $W_{M^{+}}\left(V_{M^{+}}\right)=0$ at some point $V_{M^{+}} \in\left(V_{C}, V_{S}\right)$. Lemmas 7 and 10 guarantee the existence of such an $M^{+}$, or that $\Sigma_{M^{+}} \geq 0$ on $\left(V_{C}, V_{S}\right.$ ]. In the latter case Lemma 6 guarantees the existence of such an $M^{+}$. Next choose an $M^{-}>0$ with the property that $W_{M}->0$ on the interval $\left[V_{C}, V_{S}\right]$. The existence of such an $M^{-}<M$ is guaranteed by Lemma 8 by taking $K>\sup W$ in that lemma. In fact we can take $M^{-}$such that $W_{\eta}>0$ on $\left[V_{C}, V_{S}\right]$ for all $\eta \in\left[M^{-}, M^{-}+\varepsilon\right]$ for some $\varepsilon>0$. Next, we define $S$ to be the subset of $\left[M^{-}, M^{+}\right]$in which the functions $W_{\mu}$ have zeros in $\left(V_{C}, V_{S}\right)$ :

$$
S=\left\{\mu \in\left[M^{-}, M^{+}\right]: \lim _{V \downarrow V_{\mu}} W_{\mu}=0 \text { for some } V_{\mu} \in\left(V_{C}, V_{S}\right)\right\} .
$$

This set is clearly non-empty since $M^{+} \in S$. Further, $S$ is bounded below by $M^{-}+\varepsilon$. We define

$$
\tau=\inf _{\mu \in S} \mu
$$

We now derive some important properties of $\tau$. In the following three lemmas we restrict our attention only to non-spherical models.

Lemma 11. For static non-spherical stellar models composed of fluid whose equation of state satisfies either Assumption A, Eq. (72), or Assumption B, Eq. (76), it follows that $\tau \notin S$ 
Proof. Suppose on the contrary that $\tau \in S$. Then by definition $V_{\tau}>V_{C}$ and $\lim _{V \downarrow V_{\tau}} W_{\tau}=0$. By Lemma $10 \Sigma_{\tau} \geq 0$ in this case. If $V_{\tau}$ is a regular zero of $W_{\tau}$, then the conditions of the Beig and Simon [7] theorem are satisfied and so the stellar model must be spherical. This lemma, however, is concerned only with non-spherical stars and so we conclude that $V_{\tau}$ must be an irregular zero of $W_{\tau}$. From Lemma 3 it follows then that

$$
\lim _{V \downarrow V_{\tau}} \frac{d W_{\tau}}{d V}=8 \pi V_{\tau}(\varrho+3 p) .
$$

Lemma 4, Eq. (35), shows that for any $V \in\left(V_{\tau}, V_{S}\right)$ there exists a $\delta_{\nu}>0$ such that for all $\delta \in\left(0, \delta_{\nu}\right)$,

$$
\left|W_{\tau-\delta}(V)-W_{\tau}(V)\right|+\left|\frac{d W_{\tau-\delta}}{d V}(V)-\frac{d W_{\tau}}{d V}(V)\right|<C \delta,
$$

where $C=C(V, \tau)$ is independent of $\delta$. Without loss of generality we may take $\delta_{\nu}<\varepsilon$ so that $\tau-\delta \in\left[M^{-}, M^{+}\right]$; however, $\tau-\delta \notin S$ since $\tau=\inf S$. Thus, $W_{\tau-\delta}>0$ on $\left(V_{C}, V_{S}\right]$. If $W_{\tau-\delta}$ vanished at a point $V_{\tau-\delta}$, then from Lemma 10 we would know that $\Sigma_{\tau-\delta} \geq 0$ on $\left(V_{\tau-\delta}, V_{S}\right.$ ]. The argument is more subtle, however, if $W_{\tau-\delta}$ has no zero at all. In this case we may use Eqs. (86) and (87) to compare $W_{\tau-\delta}$ with $W_{\tau}$ at $V=V_{\tau}+\varepsilon_{\nu}$ (for sufficiently small $\varepsilon_{\nu}$ ) and so conclude that

$$
\frac{d W_{\tau-\delta}}{d V}-\frac{8 \pi}{3}\left(V_{\tau}+\varepsilon_{\nu}\right)(\varrho+3 p)>0
$$

for sufficiently small $\delta$. Thus from Lemma 2 , we conclude that $\Sigma_{\tau-\delta} \geq 0$ on $\left(V_{C}, V_{\tau}+\varepsilon_{\nu}\right.$ ); and using Lemma 10 that $\Sigma_{\tau-\delta} \geq 0$ on the entire interval $\left(V_{C}, V_{S}\right.$. It follows then from Lemma 6 that $W_{\tau-\delta} \geq W$ on $\left[V_{C}, V_{S}\right]$. And so in particular that $W_{\tau-\delta}\left(V_{\tau}\right) \geq \sup _{V=V_{\tau}} W$.

Consider for a moment the properties of $\sup _{V=V_{*}} W$ for $V_{*} \in\left[V_{C}, V_{S}\right]$. This function must be strictly positive unless $V_{*}=V_{C}$. Otherwise, if there were a $V_{*}>V_{C}$ for which sup $W=0$ then we could find an open ball contained entirely in the open $V=V_{*}$

set $V<V_{*}$ whose surface intersects and is tangent to the surface $V=V_{*}$ at some point $q$. In this open ball the boundary value maximum principle applied to Eq. (2) implies that the gradient of $V$ may not vanish at the maximum point $q$, and so $W$ may not vanish there. Thus, $\sup _{V=V_{*}} W>0$.

Now since $W_{\tau-\delta}\left(V_{\tau}\right) \geq \sup _{V=V_{\tau}} W>0$ we get a contradiction with Eq. (87) if $\delta$ is taken small enough. Thus, we conclude that $V_{\tau}$ can not be in $\left(V_{C}, V_{S}\right)$ and so $\tau \notin S$.

The next lemma guarantees that functions $r_{\tau}$ and $m_{\tau}$, solutions to Eqs. (10) and (11), exist on the interval $\left(V_{C}, V_{S}\right]$ with the important property that $\lim _{V \downarrow V_{C}} W_{\tau}=0$.

Lemma 12. For static non-spherical stellar models composed of fluid whose equation of state satisfies either Assumption A, Eq. (72), or Assumption B, Eq (76), it follows that $W_{\tau}>0$ on the interval $\left(V_{C}, V_{S}\right]$, and $\lim _{V \downarrow V_{C}} W_{\tau}=0$. 
Proof. By Lemma 11, $\tau \notin S$ and so $W_{\tau}>0$ on $\left(V_{C}, V_{S}\right.$. Assume to the contrary of this lemma that $\lim _{V \downarrow V_{C}} W_{\tau} \neq 0$. It follows from Lemma 4 then that there exists a $\bar{\delta}>0$ such that for all $\delta \in(0, \bar{\delta})$ we have $W_{\tau+\delta}>0$ on $\left[V_{C}, V_{S}\right]$. But by definition $\tau=\inf S$ and by Lemma $11 \tau \notin S$. Thus there exists a small enough $\delta$ so that $\tau+\delta \in S$, and hence $W_{\tau+\delta}$ vanishes at $V_{\tau+\delta} \in\left(V_{C}, V_{S}\right.$ ]. Thus we have a contradiction unless $\lim _{V \downarrow V_{C}} W_{\tau}=0$.

Next we define $S_{C}$ to be the set of mass parameter values for which $W_{\mu}$ has a zero at the center of the physical star $V=V_{C}$ :

$$
S_{C}=\left\{\mu \in\left[M^{-}, M^{+}\right]: W_{\mu}>0 \text { on }\left(V_{C}, V_{S}\right] \text { and } W_{\mu}\left(V_{C}\right)=0\right\}
$$

This set is not empty because $\tau \in S_{C}$ as shown by Lemma 12 . Now we let

$$
\nu=\inf _{\mu \in S_{C}} \mu
$$

The following lemma shows that $\nu$ is also an element of $S_{C}$.

Lemma 13. For static non-spherical stellar models composed of fluid whose equation of state satisfies either Assumption A, Eq. (72), or Assumption B, Eq. (76), it follows that $\nu \in S_{C}$ and there exists a $\bar{\delta}>0$ such that for all $\delta \in(0, \bar{\delta})$ we have $W_{\nu-\delta}>0$ on $\left[V_{C}, V_{S}\right]$.

Proof. Assume on the contrary that $\nu \notin S_{C}$. Then for any $\delta_{1}>0$ there exists a $\delta_{2} \in\left(0, \delta_{1}\right)$ such that $\nu+\delta_{2} \in S_{C}$; thus,

$$
W_{\nu+\delta_{2}}\left(V_{C}\right)=0
$$

However, we also have $W_{\nu}\left(V_{C}\right)>0$ since $\nu \notin S_{C}$. We now show that this contradicts the continuity of $W_{\nu}$ with respect to $\nu$ guaranteed by Lemma 4 . Using the same argument given in Lemma 11 to prove that $\Sigma_{\tau-\delta} \geq 0$, it follows that $\Sigma_{\nu} \geq 0$ on $\left[V_{C}, V_{S}\right]$. Then from Lemma 6 we conclude that $W_{\nu}>W \geq 0$ on $\left[V_{C}, V_{S}\right]$. The continuity of $W_{\nu}$ guaranteed by Lemma 4 insures then that there exists a $\delta_{3}>0$ such that $W_{\nu+\delta_{4}}>0$ on $\left[V_{C}, V_{S}\right]$ for every $\delta_{4} \in\left(0, \delta_{3}\right)$. This, however, contradicts Eq. (91) if we take $\delta_{3}=\delta_{1}$. Thus we conclude that $\nu \in S_{C}$.

Next let $\nu-\bar{\delta}>M^{-}$and let $\delta \in(0, \bar{\delta})$. Since $W_{\nu}\left(V_{C}\right)=0$ from the argument above, it follows from Lemma 10 that $\Sigma_{\nu} \geq 0$ on $\left[V_{C}, V_{S}\right]$. Next, from the monotonicity of $W_{\nu}$ derived in Lemma 5, we conclude that $W_{\nu-\delta}>W_{\nu}$ on $\left(V_{C}, V_{S}\right]$. And since $\nu-\delta<\nu=\inf S_{C}$ we conclude that $W_{\nu-\delta}>0$ on the closed interval $\left[V_{C}, V_{S}\right]$ as well.

The solution to Eqs. (10)-(14) with parameter $\mu=\nu$, where $\nu$ is defined as in Eq. (90), is in effect our "reference spherical model." We will show in the following sections that any static stellar model whose equation of state satisfies the assumptions of Sect. 4 must be identical to this reference model, and in particular must satisfy $W=W_{\nu}$ with $\nu=M$. 


\section{The Conformal Factors $\psi_{\mu}(V)$}

We next introduce the function $\psi_{\mu}$ which will be used as a conformal factor to transform the spatial metric $g_{a b}$. In the exterior of the star, i.e., for $V \in\left[V_{S}, 1\right)$ we set

$$
\psi_{\mu}=\frac{1}{2}(1+V)
$$

while inside the star, $V \in\left(V_{\mu}, V_{S}\right]$,

$$
\frac{d \psi_{\mu}}{d V}=\frac{\psi_{\mu}}{2 r_{\mu} \sqrt{W_{\mu}}}\left[1-\sqrt{1-\frac{2 m_{\mu}}{r_{\mu}}}\right],
$$

with $\psi_{\mu}\left(V_{S}\right)=\left(1+V_{S}\right) / 2$. This is a positive $C^{1,1}$ function on $\left(V_{\mu}, 1\right)$. Using Eq. (32) it is straightforward to verify that $\psi_{\mu}$ also satisfies

$$
\frac{d^{2} \psi_{\mu}}{d V^{2}}+\frac{2 \pi}{W_{\mu}}\left[2 V(\varrho+3 p) \frac{d \psi_{\mu}}{d V}-\varrho \psi_{\mu}\right]=0
$$

The following lemma establishes an important property of $\psi_{\mu}$ :

Lemma 14. Assume that the equation of state $\varrho=\varrho(p)$ is a non-negative, nondecreasing, $C^{1}$ function which satisfies the inequality $5 \varrho^{2}-6 p(\varrho+3 p) \kappa \geq 0$. Then the function $\psi_{\mu}$ defined in Eqs. (92) and (93) satisfies $d^{2} \psi_{\mu} / d V^{2} \geq 0$ on $\left(V_{\mu}, 1\right)$.

Proof. For the case when $V_{\mu}$ is a regular zero of $W_{\mu}$ the proof follows almost exactly the proof of Lemma 1 of Masood-ul-Alam [4]. The proof when $V_{\mu}$ is an irregular zero is very similar as well. Note that $\psi_{\mu}^{2}$ is called $\Omega$ in that reference. To generalize the proof to include the case when $V_{\mu}$ is an irregular zero of $W_{\mu}$ we define

$$
f_{\mu}=8 \pi \varrho-16 \pi(\varrho+3 p) \frac{V}{\psi_{\mu}} \frac{d \psi_{\mu}}{d V} .
$$

Differentiating $f_{\mu}$ and using Eq. (94) it follows that

$$
\begin{aligned}
16 \pi V(\varrho+3 p) \frac{d f_{\mu}}{d V}=f_{\mu}^{2} & -\left[48 \pi \varrho+16 \pi(\varrho+3 p) \kappa+64 \pi^{2} V^{2}(\varrho+3 p)^{2} W_{\mu}^{-1}\right] f_{\mu} \\
+ & 64 \pi^{2}\left[5 \varrho^{2}-6 p(\varrho+3 p) \kappa\right] .
\end{aligned}
$$

This equation implies that if $f_{\mu}(V)<0$ for some $V \in\left(V_{\mu}, V_{S}\right)$ we have $d f_{\mu} / d V>0$ at that $V$. But $\lim _{V \downarrow V_{\mu}} f_{\mu}(V)=0$ if $V_{\mu}$ is a regular zero and $\lim _{V \downarrow V_{\mu}} f_{\mu}(V)=+\infty$ if $V_{\mu}$ is an irregular zero. The last limit follows from Eq. (93) and Lemma 3 since $m_{\mu}$ becomes negative near $V_{\mu}$ for an irregular zero. So $f_{\mu}$ is not negative at $V=V_{\mu}^{\mu}$ and cannot become negative for larger values of $V$ unless $d f_{\mu} / d V$ is negative there. But this contradicts Eq. (96), so we conclude that $f_{\mu}(V) \geq 0$, and hence by Eq. (94) above $d^{2} \psi_{\mu} / d V^{2} \geq 0$. 


\section{The Main Theorem}

We will now consider the conformal factor $\psi_{\nu}$ with $\nu$ defined in Eq. (90). In order to be useful as a conformal factor for the physical metric $g_{a b}, \psi_{\nu}$ must satisfy certain minimal smoothness conditions when considered as a function on the physical threedimensional constant- $t$ hypersurfaces. From its definition in Eqs. (92) and (93) it follows that $\psi_{\nu}$ is a $C^{1,1}$ function of $V$ for $V_{C}<V<1$. Thus $\psi_{\nu}$ will be a $C^{1,1}$ function of position everywhere, except possibly at $V=V_{C}$, because $V$ is a $C^{1,1}$ function of position by virtue of the fact that it is a solution of Eq. (2). Using Eq. (93) the gradient of $\psi_{\mu}$ (for any $\mu$ ) can be expressed in the form

$$
D_{a} \psi_{\mu}=\frac{\psi_{\mu}}{2 r_{\mu} \sqrt{W_{\mu}}}\left[1-\sqrt{1-\frac{2 m_{\mu}}{r_{\mu}}}\right] D_{a} V .
$$

The coefficient of $D_{a} V$ is bounded at a regular zero of $W_{\mu}$ and so $D_{a} \psi_{\mu}$ vanishes there. Thus $\psi_{\nu}$ is a $C^{1,1}$ function of position everywhere in this case. Unfortunately in case $V_{\nu}$ is an irregular zero, then Eq. (97) and Lemma 3 imply that $D_{a} \psi_{\nu}$ is not continuous at the level set $V=V_{C}$ in the physical three-space. However by virtue of Lemma 13, $W_{\nu-\delta}\left(V_{C}\right)>0$, where $\delta$ is as in that lemma. Hence from Eq. (97) it follows trivially that $\psi_{\nu-\delta}$ will be a $C^{1,1}$ function of position in the physical threespace. By choosing $\delta$ small enough we can also ensure that $d^{2} \psi_{\nu-\delta} / d V^{2} \geq 0$ on $\left[V_{C}, V_{S}\right]$. This is because we have from Eqs. (93) and (95) that $\lim _{V \downarrow V_{C}} f_{\nu}(V)=+\infty$. That implies there exists a $\delta$ such that $\lim _{V \downarrow V_{C}} f_{\nu-\delta}(V)>0$; this is because by Lemma 13, $W_{\nu-\delta}\left(V_{C}\right)>0$, and by Eq. (93) $d \psi_{\nu-\delta} / d V$ is bounded at $V_{C}$. Thus Lemma 14 gives $f_{\nu-\delta}(V) \geq 0$ and so $d^{2} \psi_{\nu-\delta} / d V^{2} \geq 0$ on $\left[V_{C}, V_{S}\right]$. In the main theorem that follows we apply the positive mass theorem to the conformal metric $\bar{g}_{a b}=\psi^{4} g_{a b}$, where

$$
\psi= \begin{cases}\psi_{\nu} & \text { if } V_{C} \text { is a regular zero of } W_{\nu} ; \\ \psi_{\nu-\delta} & \text { if } V_{C} \text { is an irregular zero of } W_{\nu} .\end{cases}
$$

Our preparations are now complete. We can now combine the results of the previous sections into a complete proof of our main result

Theorem. Consider a static stellar model as defined in Eqs. (1)-(6) having an equation of state which satisfies Condition A, Eq (72), or Condition B, Eq. (76). Then this stellar model must be spherically symmetric.

Proof. We define the conformal metric

$$
\bar{g}_{a b}=\psi^{4} g_{a b}
$$

where $\psi$ is defined in Eq. (98). The mass associated with $\vec{g}_{a b}$ is zero as a consequence of the definition of $\psi$ in the exterior of the star, Eq. (92), and the asymptotic boundary conditions on the metric functions, Eqs. (5) and (6). The scalar curvature associated with $\bar{g}_{a b}$ is easily evaluated, using Eqs. (2) and (3) with the result,

where $\tilde{W}$ is defined as

$$
\bar{R}=(\tilde{W}-W) \frac{8}{\psi^{5}} \frac{d^{2} \psi}{d V^{2}}
$$

$$
\tilde{W}= \begin{cases}W_{\nu} & \text { if } V_{C} \text { is a regular zero of } W_{\nu} ; \\ W_{\nu-\delta} & \text { if } V_{C} \text { is an irregular zero of } W_{\nu} .\end{cases}
$$


It follows from Lemmas 12 and 13 that $\tilde{W}>0$ on $\left(V_{C}, V_{S}\right.$ ]. Then as a consequence of Lemmas 5 and $6, W_{\nu} \geq W$ when $V_{C}$ is a regular zero of $W_{\nu}$ and $W_{\nu-\delta}>W_{\nu} \geq W$ when $V_{C}$ is an irregular zero of $W_{\nu}$. Next, Conditions $\mathbf{A}$ or $\mathbf{B}$ on the equation of state imply via Lemma 9 that $5 \varrho^{2}-6 p(\varrho+3 p) \kappa \geq 0$. Thus Lemma 14 implies that

$$
\frac{d^{2} \psi}{d V^{2}} \geq 0
$$

Therefore the scalar curvature $\bar{R}$, Eq. (100), is non-negative. As the discussion following Lemma 14 demonstrated, the conformal factor $\psi$ and hence the metric $\bar{g}_{a b}$ are $C^{1,1}$ functions of position on the three-dimensional constant- $t$ hypersurfaces. Thus Bartnik's [23] version of the positive mass theorem (Schoen and Yau [24]) may be applied. Since $\bar{R} \geq 0$ while $\bar{g}_{a b}$ has zero mass, the metric $\bar{g}_{a b}$ must in fact be flat. Hence the physical metric $g_{a b}$ is conformally flat. It follows then from the arguments given by Lindblom [9] (based on earlier work of Avez [25] and Künzle [26]) that the static stellar model must be spherically symmetric.

Although the conclusion of spherical symmetry is our primary interest, the argument given above in fact establishes more. It demonstrates the uniqueness of the static stellar model having surface potential $V_{S}$ and satisfying the assumptions on the equation of state in Sect. 4. In particular the vanishing of $\bar{R}$ in Eq. (100) implies that $W=\tilde{W}$. This equality is impossible for the irregular zero case (see Lemma 13). We conclude that the center of the star must coincide with a regular zero of $W_{\nu}$, and that $W=W_{\nu}$ with $\nu=M$. This argument rules out the possibility of multiple spherical models having the same surface potential $V_{S}$ for stars whose equation of state satisfies these conditions.

\section{Concluding Remarks}

The conditions placed on the equation of state in Sect. 4 are almost certainly too restrictive, although they do include large open sets in the space of all equations of state. We found it necessary to use these conditions, however, to show in our proof that the functions $\Sigma_{\mu}$ were positive and that the conformal functions $\psi_{\mu}$ had positive second derivatives. How can our proof be modified to make those conditions on the equation of state unnecessary? Perhaps there is some more elegant way than Eqs. (92) and (93) to choose a conformal factor which makes both scalar curvature nonnegative and mass zero directly. While some alternate construction might bypass the need to constrain the signs of $\Sigma_{\mu}$ and $d^{2} \psi_{\mu} / d V^{2}$, at present we do not know of a better choice. In our proof the nonnegativity of $\Sigma_{\mu}$ is used to prove the monotonicity of $W_{\mu}$ (in $\mu$ ) as well as to prove that $W_{\mu}-W$ is non-negative. Perhaps the need for the strict non-negativity of $\Sigma_{\mu}$ can be avoided by analyzing the eigenvalues of the operators on the left sides of Eqs. (40) and (44). These eigenvalues may be related to the existence of multiple solutions of the spherical equations all having the same value of $V_{S}$. And, perhaps a more powerful version of the maximum principle can be used which allows $\Sigma_{\mu}$ to have somewhat negative values; perhaps down to a minimum which is controlled by the magnitude of the first negative eigenvalue.

The method of proof used up to now establishes that the static spherical stellar model having a given surface potential $V_{S}$ is the unique static model having that $V_{S}$. It would be interesting to know what conditions on the equation of state are the minimal ones needed to guarantee this absolute uniqueness. We do not believe that the equations of state allowed by Condition $\mathbf{A}$ together with with those allowed by $\mathbf{B}$ are the largest such set. We also know that there exist physically reasonable equations 
of state in which more than one spherical model exists for a given value of $V_{S}$. Thus, in order to extend the proof to include these equations of state it will be necessary to find a criterion to decide which one of the multiple spherical models should be used as the reference model for a given static model.

We now have a better understanding of the condition appearing in Lemma 14. If this condition is violated everywhere, we have shown [8] that the stellar model cannot have finite size, although it must nevertheless have spherical symmetry. There exist static stellar models with equations of state that violate this condition locally, e.g., stars based on "realistic" neutron-star equations of state. We expect that all such models must be spherically symmetric as well, but the question of how to extend the proof in these cases is presently unresolved.

Acknowledgements L.L. thanks Jim Isenberg and the Institute of Theoretical Science of the University of Oregon for their hospitality during a visit in which a portion of this research was completed We also thank R. Beig and W. Simon for commenting on a preliminary version of this paper. This research was supported by grants PHY-9019753, AST-9114925, and PHY-9012301 from the National Science Foundation, and grant NAGW-2951 from the National Aeronautics and Space Administration.

Note added in proof. Present address: A. K. M. Masood-ul-Alam, Institut for Advanced Studies in Basic Sciences, Zanjan, Iran.

\section{References}

1. Lichtenstein, L.: Sitzungsber Preuss. Akad Wiss., Phys Math. Kl, 1120 (1918)

2. Lindblom, L. Phil.: Trans. Roy. Soc. Lond A, 340, 353-364 (1992)

3. Masood-ul-Alam, A.K M.: Class. Quantum Grav. 4, 625-633 (1987)

4. Masood-ul-Alam, A.K.M.: Class. Quantum Grav. 5, 409-421 (1988)

5. Lindblom, L.: J. Math. Phys. 29, 436-439 (1988)

6. Beig,R, Simon, W : Lett. Math. Phys. 21, 245-250 (1991)

7. Beig, R., Simon, W.: Commun. Math. Phys 144, 373-390 (1992)

8 Lindblom, L, Masood-ul-Alam, A K.M.: In: Directions in general relativity II Essays in Honor of Dieter Brill. B.L. Hu, T.A. Jacobson (eds ) London: Cambridge University Press, 1993, pp. 172-181

9. Lindblom, L.: J Math. Phys 21, 1455-1459 (1980)

10 Masood-ul-Alam, A.K M.: Commun. Math Phys 108, 193-211 (1987)

11 Beig, R.: Gen Rel. Grav. 12, 439-451 (1980)

12 Masood-ul-Alam, A.K.M : The topology of asymptotically Euclidean static perfect fluid spacetime. Ph. D. Thesis, Australian National University (1985)

13 Bunting, G.L, Masood-ul-Alam, A K.M.: Gen. Rel Grav 19, 147-154 (1987)

14 Hartman, P : Ordinary differential equations. Boston: Birkhäuser 1982

15 Lindblom, L: Ap J 278, 364-368 (1984)

16 Coddington, E A., Levinson, N.: Theory of ordinary differential equations New York: McGrawHill 1955

17. Gilbarg, D, Trudinger, N.S : Elliptic partial differential equations of second order Berlin, Heidelberg, New York: Springer 1983

18. Robinson, D : Gen. Rel. Grav. 8, 695-698 (1977)

19 Bondi, H : Proc. Roy. Soc A 282, 303-317 (1964)

20. Rendall, A D, Schmidt, B D.: Class Quantum Grav 8. 985-1000 (1991)

21 Simon, W : Class Quantum Grav 10, 177-181 (1993)

22 Buchdahl, H. A.: Phys Rev 116, 1027-1034 (1959)

23 Bartnik, R : Commun Pure Appl. Math 39, 661-693 (1986)

24. Schoen, R, Yau, S -T.: Commun Math Phys 65, 45-76 (1979)

25 Avez, A : Ann Inst Henri Poincaré A 1, 291-300 (1964)

26 Künzle, H P : Commun Math Phys. 20, 85-100 (1971)

Communicated by $S-T$ Yau 
